\title{
A NOVEL DBMD IMPLEMENTATION FOR BIG DATA MINING AND CLUSTERING VIA CLOUD COMPUTING
}

\author{
A. Vaitheeswari \\ Student, Department of Computer Science and Engineering, P. S. R. Engineering College, \\ Sivakasi, 626140, Tamil Nadu, India \\ Dr. N. Krishnaveni \\ Assistant Professor, Department of Computer Science and Engineering, P. S. R. Engineering College, \\ Sivakasi, 626140, Tamil Nadu, India
}

\begin{abstract}
Matrix structure was one of the most important devices for finding data from big data. Here you'll find data produced by current applications using cloud computing. However, moving big data using such a system in a performance computer or through virtual machines is still inefficient or impossible. Furthermore, big data is often gathered data from a variety of data sources and stored on a variety of machines using scheduling algorithms. As a result, such data usually bear solid shifted commotion. Growing circulated matrix deterioration is necessary and beneficial for big data analysis. Such a plan should have a good chance of succeeding. Represent the diverse clamour and deal with the correspondence problem in a disseminated manner. In order to do this, we used a Bayesian matrix decay model (DBMD) for big data mining and grouping. Only three approaches to disseminated computation are considered: 1) accelerate slope drop, 2) alternating path method of multipliers (ADMM), and 3) observable derivation. We look at how these approaches could be mixed together in the future. To deal with the commotion's heterogeneity, we suggest an ideal module weighted norm that reduces the assessment's differentiation. At Finally, a comparison was made between these approaches in order to understand the differences in their outcomes.
\end{abstract}

Keywords: Bayesian Matrix Disintegration; Big Data; Cloud Computing; Data Mining; Grouping; Distributed Calculation.

\section{Introduction}

As the cloud advances to handle large-scale applications like SAAS (Software as a Service), PAAS (Platform as a Service), and so on, there has been a surge in interest to move mining operations to the cloud. Cloud Computing improves the efficacy and economy of distributing assets on demand. It also allows the examination provider to keep costs down and optimize returns beyond what a pay-per-use model would provide. Effective technology management and high-stage cloud management can be analyzed as the reasonable organization of private cloud architecture [1]. Clouds contain emerge as a calculate communications so as to allow intended for the fast let go of compute possessions as necessary in a virtualized, scalable manner [4]. In the cloud computing environment, virtual machines advancements be constantly life form second-hand to provide, link, plus perfect preparations. Data mining entail combining the discovery of important models plus relations so as to be able to exist concealed in incredibly big database [3]. Cluster is a novel technique for locating emitted data and hidden relationships. The most common bunch analysis is distance support clustering. Two common methods are hierarchical gathering and panel clustering. The hierarchical clustering approach involves arranging partitions in such a way that each partition is established keen on the after that divider inside the series. The divider cluster method divides the record eager on a shape of classes [8]. The representative aid clustering strategy is a method for partitioning a group of bodies into various predictable assortments that are similar. The K-Means algorithm and the K-Mediod method be two commonly used partition clustering methods [9]. In this paper, we propose in the direction of expand a data mining move toward known as HVKM - Hierarchical Virtual K-Means Approach for a client who wants to overhaul their commerce psychoanalysis. We know where data from our sphere of notice is being concentrated inferable from its function as a segment supporting PAAS (Platform as a Service), SAAS (Service as a Service) (Software as a Service). In this paper, we attempt to assemble certain problem 
areas for the purpose of elimination using Hierarchical k-means to obtain commerce payment in domain such as CRM (customer relationship management), POS (point of sale), international stock market association, regulatory health trends. For example, mining results would assist the business forecaster in analyzing the item bunch in the market and the demand for manufactured goods due to CRM and POS. Disseminated computing, utility computing, network computing, and concurrent computing are all examples of cloud computing. Cloud computing is still in its early stages of research and implementation, and it has yet to form a unified standard and context. Cloud computing most important feature is the ability to provide large-scale storage and computing, as well as services related to virtualization information. These types of cloud computing allow data to be analyzed, stored, and cycled.

\section{Related Works}

\subsection{Software-Based Power Efficiency}

In a software-based approach, the pre-owned force may be limited by the implemented scheduling measure. Customers' requests for services are assigned to Virtual Machines that can present them under the parameters of the SLA (Service Level Agreement). To achieve the goal of lowering the level of force consumption, the scheduling procedure selects Virtual Machines (VMs) that run on items machines (workers) and consume low force. Many expected methods depend on the use of software or scheduling [5]. [7], for example A rack-aware scheduling approach was implemented with the aim of reducing service time, computing asset power, and noncomputing asset force. The procedure is based on the hereditary method. The authors of [8] used a flexible scheduling algorithm to determine the trade-off between the client's need for time and the provider's need for energy utilization. To do this, a change parameter is changed to zero in on reducing the time or lowering the amount of energy used. M. Kumar and S. Sharma [9] proposed a scheduling approach focused on Particle Swarm Optimization to reduce both time and monetary costs by limiting the amount of energy consumed by data community staff. They've devised a mathematical model for holding allocation based on a given health task. They also pay attention to the tasks' deadlines as a quality-of-service function centered on the specified wellness job. In [10], a scheduling algorithm was implemented that considers the arranging of solicitations based on the type of workload of Virtual Machines VMs in homogeneous clouds. It's possible that the workload is CPU or I/O bound. The algorithm's main aim is to reduce the amount of electrical energy burned by and service level agreement breaches.

\subsection{Efficacy of the Force Based on the Restructuring Method}

To restrict the degrees of force used, the consolidation method reduces the number of active personnel. The staffs who have owed Virtual Machine VMs are tested in this method, and some of them are chosen to be dosed based on their utilization rate. The solicitations assigned to the VMs organization on resting workers are replaced and migrated to a few selected Virtual Machine VMs on active workers. The consolidation technique is seen in many executed approaches [15]. The writers of [16] have taken care to consider the dependability of physical jobs while enticing consolidation options. They used a Markov chain model to represent worker dependability in the cloud. Their method selects the intended worker based on dependability and force use. AlDulaimy et al. [17] implemented a consolidation-based strategy that considers the types of occupations that are assigned to Virtual Machine VMs before deploying them. For the initial development of Virtual Machine VMs and the placemat of the migrated Virtual Machine VMs, the method relies on the Multiple-Choice Problem. If necessary, the method migrates Virtual Machine VMs of dissimilar occupation styles to the same worker. M. Yavari et al. [8] suggested a consolidation-based method that considers temperature and energy together. The authors are concerned about reducing the amount of heat emitted by workers while also increasing their efficiency. Their strategy is based on the Firefly Optimization approach. E. Arianyan and H. Taheri [9] proposed a multi-criteria approach for selecting VMs for migration. Memory, CPU, and bandwidth are among the parameters, while the means describe the asset's weight. The authors of [10] performed a consolidation-based draw close that took into account both the network structure and the cooling devices. Still, jobs, ventilation, and networking devices are turned off to reduce energy consumption. Their strategy includes two passes of Virtual Machine VMs allocation, with new Virtual Machine VMs and overworked Virtual Machine VMs in mind. The authors suggested consolidation-based approaches in [18]. The best match declining solution is used in both procedures. Staff with the lowest force utilization is chosen for consolidation in the main process, called ECTC enhanced-Conscious Task Consolidation.

\section{Cloud Data Mining Implementation}

Environmental factors computational data processing is used for business planning in the cloud, which does not always contribute to data mining [14]. More than a few factors contain caused the shift away from traditional or centralized data mining. When mining is done on publicly available data, the presentation issues of data mining 
demand are numerous, and the addition is absurd and exorbitant. Circulated data mining provides a basis intended for scalability, allowing the separation of large datasets utilizing elevated dimensionality keen on lesser subsets to need computational resources on their own [13]. Data dealing out is a component of the HVKM method, which also includes data circulation and alteration. Though, data sharing is a difficult challenge when dealing with a regionalized major database. As the demand for data breakdown grows, a single data hub would be unable to process all of the recovered data promptly. To maintain the above condition, virtualization ideas for data preparation and computational assets are presented [7]. Data mining is carried out using a variety of workers who are used to distribute data through dissimilar virtual machines (VMs). Cluster is a technique of compiling a position of bodily or theoretical articles into a program of similar substance [14]. K-means is the majority common knowledge algorithm of all clustering methods. The top-down or granular perspective can be used for hierarchical clustering. We believe in $k$ groups and split them into $k+1$ bunches in a top-down move toward.

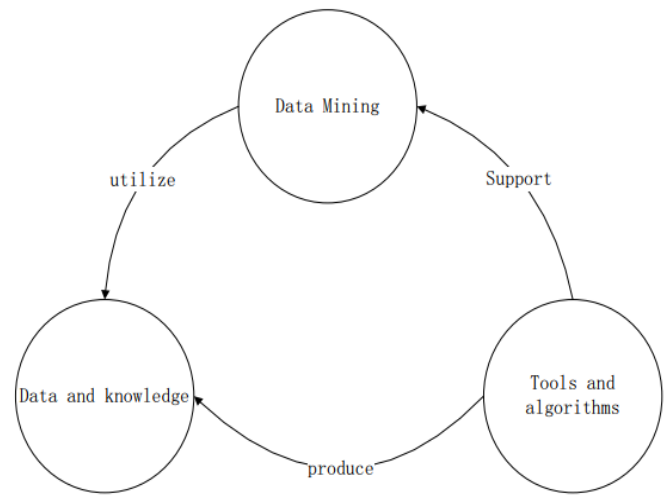

Fig. 1. The constituent elements of the data mining model

Data mining workflow includes data collection, data mining, data pre-processing, assessment, model analysis, and implementation, all of which are following the general methodology of data mining. We join the k group into k-1 in the base-up approach. The objective is to combine the two groups into a new one by selecting the most similar bunches in command. For the sifted yield, HVKM determination employs the channels in the direction of obtaining the momentary consequence and the interaction will be continuous. In the virtual climate, the system is replicated until the optimal pattern or yield is reached. After the data sifting method is applied to the passing yield, bunches, and centroids are shaped frequently during the time spent having the desired yield, which is referred to as close groups and virtual centroids. The income is iterated awaiting a characterized condition is met or the groups' consistency no longer becomes self-absorbed. The HVKM method is given a predetermined number of data sources, and the development is often base on the prototype corresponding request otherwise the data unevenness. Wherever the filter interaction is performed, HVKM's operation is iterative. Leave $\mathrm{N}$, the number of elements, alone. Everyone is made up of $\mathrm{D}=\mathrm{di} / \mathrm{i}=1 \ldots . . . \mathrm{n}$ quality, with $\mathrm{X}=\mathrm{xi} / \mathrm{i}=1 \ldots \mathrm{n}$ being each $\mathrm{D}$ info.

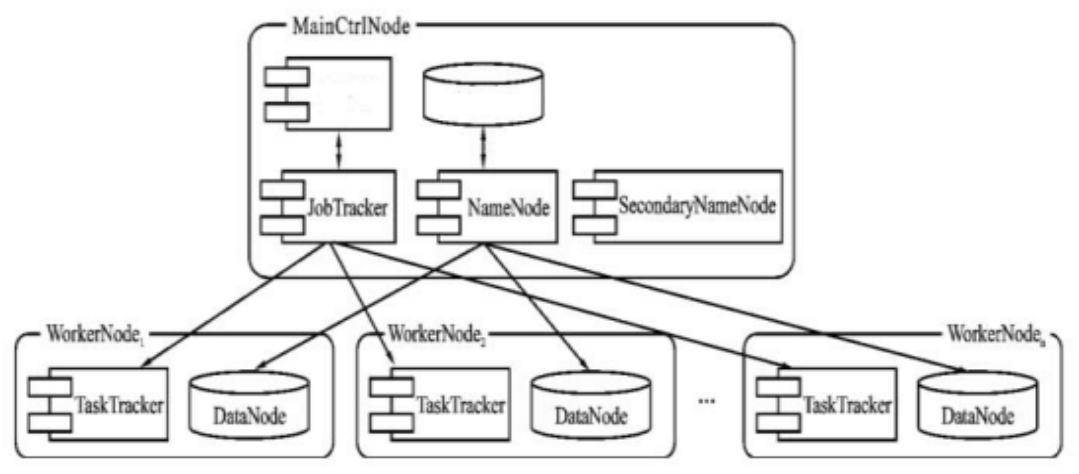

Fig. 2. Hadoop Architecture

Everyone is made up of $D=d i / i=1 \ldots . . n$ quality, with $X=x i / i=1 \ldots n$ being each $D$ info. We suggest a data mining strategy focused on cloud computing innovation, and the whole framework is open to the public. The organization's hubs are divided into two categories: key control center and worker hub. There is only one Main Control Node, which is completed by Name Node, JobTracker, data warehouse, Secondary Name Node, data 
mining system library, and data mining method library. In this system, there are multiple Worker Nodes, each of which is made up of Task Tracker and Data Node.

Let $\mathrm{K}$ be the number of necessary classes, and $\mathrm{Ci}, 1 \mathrm{I} \mathrm{K}$, be the bunch location. In n-dimensional space, each unit is discussed. $\mathrm{F}$ is the number of channels completed. We use a variety of methods in HVKM to track down the initial points. There are a few different distance scales that are used to judge distances, such as Manhattan and Euclidian. We'll use Euclidian measurements to try out a sample structure that's appealing to this tool.

If $\mathrm{p}=(\mathrm{p} 1, \mathrm{p} 2, \ldots \mathrm{pn})$ and $\mathrm{q}=(\mathrm{q} 1, \mathrm{q} 2, \ldots \mathrm{qn})$ are two ends, then $\mathrm{d}(\mathrm{p}, \mathrm{q})=\mathrm{d}(\mathrm{q}, \mathrm{p})=(|\mathrm{p} 1-\mathrm{q} 1| 2+|\mathrm{p} 2-\mathrm{q} 2| 2+\ldots+\mid \mathrm{pN}-$ $1-\mathrm{qN}-1|2+| \mathrm{pN}-\mathrm{qN} \mid 2$ ) $1 / 2$ is the detachment from q to $\mathrm{p}$, or from $\mathrm{p}$ to The HVKM execution strategy can be divided into seven levels. The number of groups necessary intended for the job is first depicted inside the kmeans system. The coldness dimension flanked by every example, inside a known category, in the direction of their bunch guided, is expected in the second phase of the k-means process. The yield of the basic method is obtained in the third step, and the yield is channeled, and novel groups are formed based on the separated results. The separating steps are maintained until the yield data is reasonably accurate.

Large-scale execution projects for a cloud level 0 hub.

(1) At levels 0 to $\mathrm{N}$, represent the $\mathrm{N}$ number of hubs and the number of iterations $\mathrm{I}$.

(2) Virtual machines VM $=\mathrm{Vm} 1 \ldots$ Vmn is owed at level 0 and Node 1.

(3) The number of groups $\mathrm{VC}=\mathrm{vci} / \mathrm{i}=1 . . \mathrm{n}$ is shaped, and the $\mathrm{k}$-means approach works on the bunches.

(4) The range move in the direction of is used to find the initial centroids.

$((p m a x-p m i n) / k) * n$ VCj $=((p m a x-p m i n) / k) * n$ VCj $=((p m a x-p m i n$ $((\mathrm{qmax}-\mathrm{qmin}) / \mathrm{k}) * \mathrm{n}=\mathrm{VCi}$

(5) The current centroid is VC (vci, vcj), with the p and q consistency standards discussed in (1) and (2). As mention above, $\mathrm{k}$ is the numeral of bunches, and $\mathrm{j}$, $\mathrm{I}$, and $\mathrm{n}$ range from 1 to $\mathrm{k}$, where $\mathrm{k}$ is a whole number. The value (pmax, pmin) represents the $\mathrm{p}$ attribute's variety, while the value (qmax - qmin) represents the q attribute's variety. Find the hold using either the Euclidean distance system or the metric system. Create the panel by assigning each model to the closest group based on these distances currently, the interval between $\mathrm{I}$ and $\mathrm{j}$ is $\mathrm{d}(\mathrm{Pi}, \mathrm{Pj})$. $\mathrm{Pi}$ and $\mathrm{Pj}$ are the properties of a substance, where I and $\mathrm{j}$ are integers ranging from 1 to $\mathrm{N}$, and $\mathrm{N}$ is the total number of properties of a known object. The numbers $\mathrm{I}, \mathrm{j}$, and $\mathrm{N}$ are all numbers. Here is where the primary results are arranged. Consider it like a VCR.

(6) A bunch of segregated outcomes is obtained at Node 1 i.e. N1 known as VF1 using a thing-based joint sifting algorithm on VCR17. A new centered is predicted based on the Filtering outcome, and the virtual bunches are framed in a hierarchical technique utilizing the base awake move toward. In this way, the procedure is frequent waiting the preferred yield is precise in some luggage and sharps sufficient in others.

\section{Results}

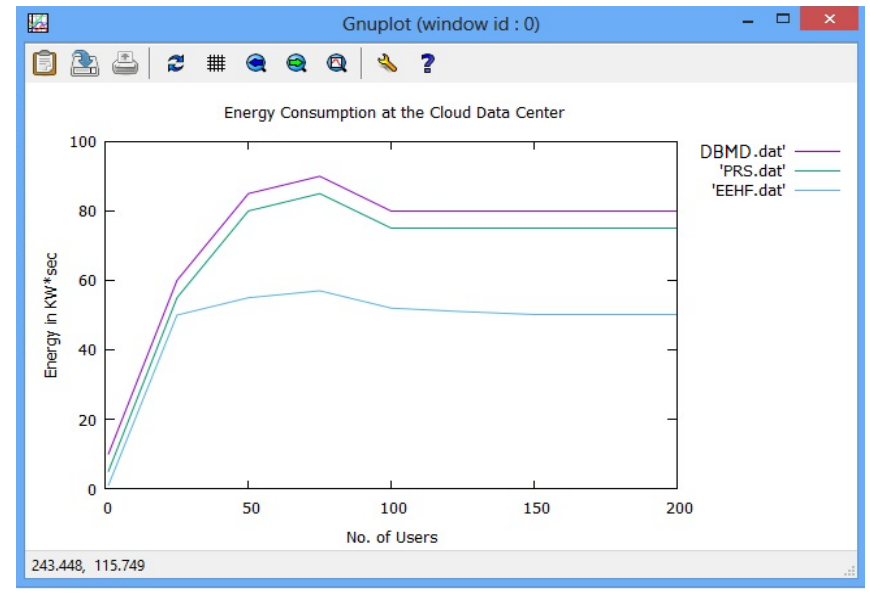

Fig. 3. Energy Consumption and Average Resources Utilization

The energy use and average asset consumption at the cloud data focus are shown separately in Fig. Since we set the active upper edge value by computing the median absolute deviation and bury the quartile range of past data separately, our proposed FFD dependent algorithm uses less energy than Inter Quartile Range (IQR) and random Virtual Machines VMs migration. Furthermore, by manipulating the bury quartile collection of 
historical data separately; the authors set the upper limit consumption of each PM. In this vein, the creator of the IQR method simply considered the CPU use of the PM to locate the overloaded PM. However, no decision was made on the switches' power consumption at the cloud data center. Furthermore, we migrated the Virtual Machine VMs from underutilized PMs to non-underutilized PMs using an arbitrary Virtual Machine VMs migration process, so we didn't choose the most appropriate PMs for the Virtual Machine VMs migration. PMs and fastens power consumption is higher as a result of random Virtual Machine VM migration.

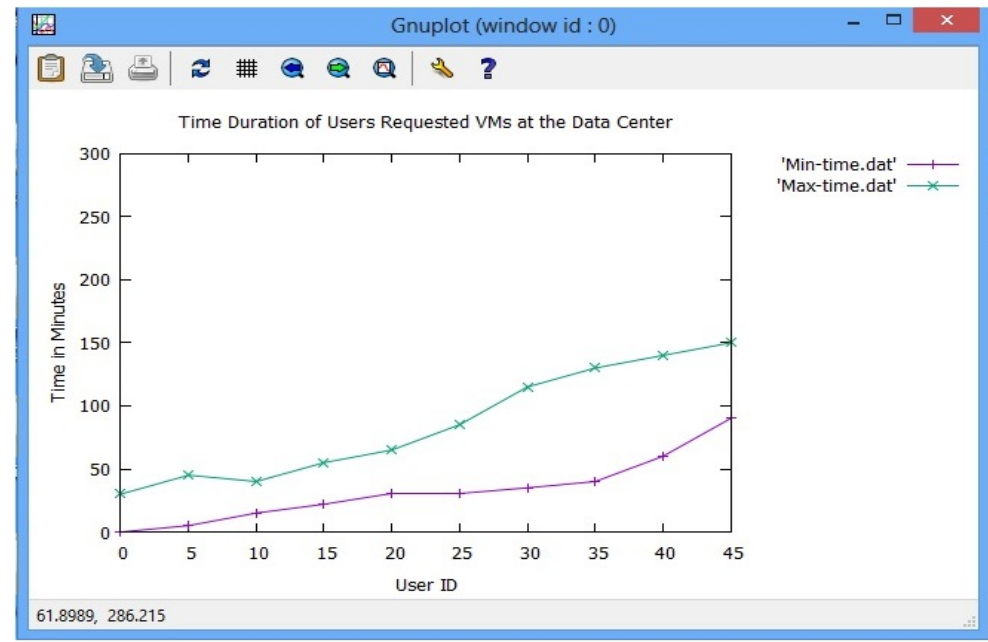

Fig. 4. Throughput comparison

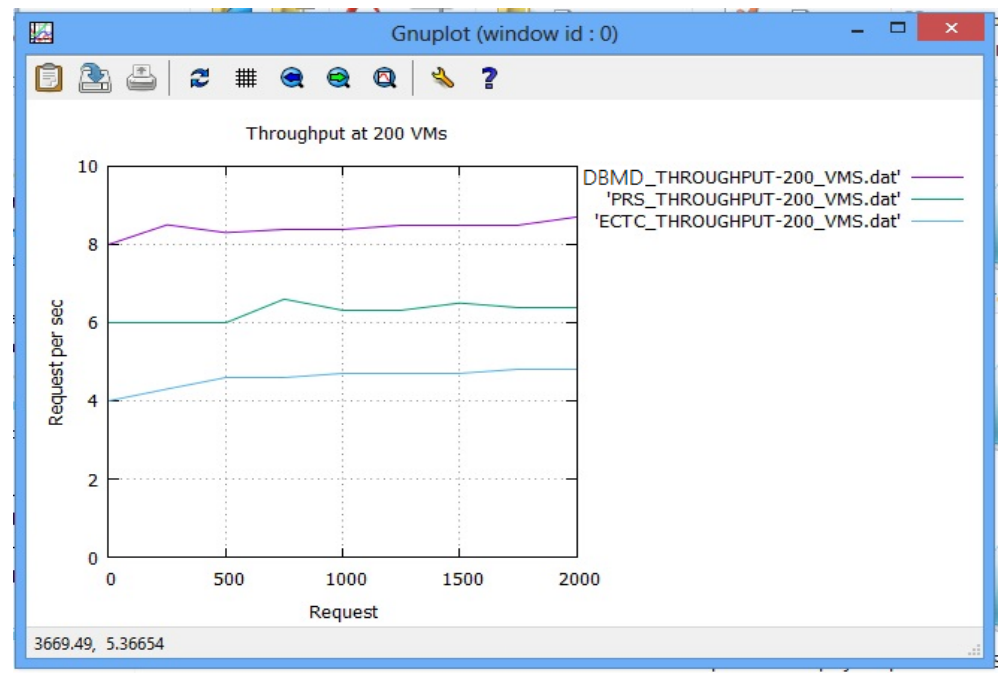

Fig. 5. Min-Time Max-Time Comparison

We estimated that each client will need between 1 and 200 Virtual Machine VMs from the service provider. The client specified a period or life season of the Virtual Machine VMs between 30 and 200 minutes in their request. At the cloud data focus, we will now allocate the clients' demand Virtual Machine VMs using the appropriate First-Fit approximation algorithm. After that, we used our proposed Virtual Machine VMs migration algorithm to transfer Virtual Machine VMs from underutilized PMs to energy-efficient PMs at the cloud data center regularly. Furthermore, the Virtual Machine VMs migration measure necessitates an additional slide in terms of force consumption. As a result, we used a consistent migration cost for each type of Virtual Machine VM, such as 10 watts (small), 20 watts (medium), 30 watts (large), and 40 watts (extra large) (x.large). 


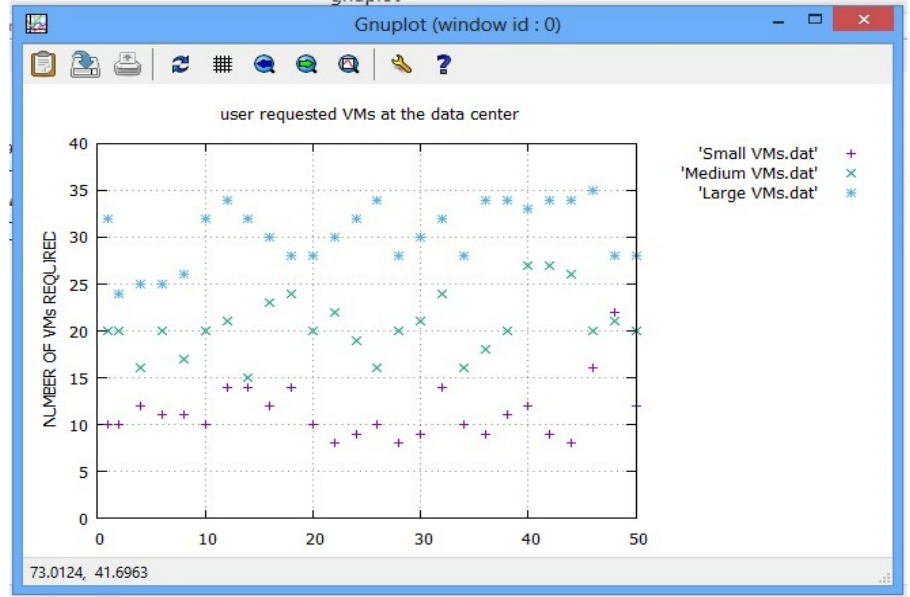

Fig. 6. No of VMs Required

\section{Conclusion}

This research work plans and evaluates a hybrid system for green cloud computing that takes into account the time-sensitive force usage model. The proposed process, in comparison to the next method proposed in the literature, uses both the turn of events and restructuring methods. Client demands are first and foremost organized according to the desires of both the force utilization and the service time. Then, using a planned scheduling process, each solicitation is assigned to the most suitable Virtual Machines VMs that can support it. Following that, a planned consolidation method is used to determine the employees who will be joined and which workers will be given Virtual Machines (VMs) of the merged workers. Finally, to perform the migration of the Virtual Machines VM from the union workers, an immigration approach is used. In terms of PUE Power Use Effectiveness, DCEP Data Center Energy Productivity, throughput, average execution time, and cost-saving, the EEH architecture outperforms approaches that rely on only one step to reduce power consumption. Collapse implications for the quantities of used force will be considered in future studies. Deep learning techniques [10], [12] can be used to incredibly predict staff utilization and learn various related scheduling and consolidation limitations. We also plan to strengthen the scheduling system by implementing a load balancing approach.

\section{References}

[1] L. Nieuwenhuis, M. Ehrenhardc and L. Prausec, "The shift to Cloud Computing: The impact of disruptive technology on the enterprise software business ecosystem,” Technological Forecasting \& Social Change, Vol. 129, 2018, pp. 308-313.

[2] M. H. Ghahramani et al., “Toward Cloud Computing QoS Architecture: Analysis of Cloud Systems and Cloud Services,” IEEE/CAA Journal of Automatica Sinica, Vol. 4, No. 1, Jan. 2017, pp. 5-17. zettabytes-by-2025/\#6edb93895459. (14 Dec. 2019)

[3] A. Shehabi, S. Smith, E. Masanet and J. Koomey, "Datacenter growth in the United States: decoupling the demand for services from electricity use,” Environmental Research Letters, Vol. 13, No. 12, 2018.

[4] M. Usman, A. Ismail, G. Salaam, H. Chizari, O. Kaiwartya, A. Gital, M. Abdullahi, A. Aliyu and, S. Dishing, "Energy-efficient Nature-Inspired techniques in Cloud computing data centers,” Telecommunication Systems, Vol. 71, 2019, pp. $275-302$.

[5] N. Jones, "How to stop data centers from gobbling up the world's electricity," Nature, Vol 561, Sep. 2018, pp. 163-166.

[6] S. Mustafa et al, "SLA-Aware Best Fit Decreasing Techniques for Workload Consolidation in Clouds," IEEE Access, Vol. 7, 2019, pp. $135256-135267$.

[7] Q. Wu, et al., "Energy and Migration Cost-Aware Dynamic Virtual Machine Consolidation in Heterogeneous Cloud Datacenters,” IEEE Transactions on Services Computing, Vol. 12, No. 4, 2019, pp. $550-563$.

[8] R. Yadav et al, "MuMs: Energy-Aware VM Selection Scheme for Cloud Data Center," Proc. of 28th International Workshop on Database and Expert Systems Applications, 2017, pp. 132-136.

[9] S. Madni, M. Latiff, Y. Coulibaly, and S. Abdulhamid, "Recent advancements in resource allocation techniques for cloud computing environment: A systematic review,"' Cluster Comput., vol. 20, pp. 2489-2533, Sep. 2017.

[10] F. Juarez, J. Ejarquea and R. M. Badiaa, "Dynamic energy-aware scheduling for parallel task-based application in cloud computing," Future Generation Computer Systems, Vol. 78, Part 1, January 2018, pp. 257-271.

[11] S. Teodoro et al, “A comparative study of energy-aware scheduling algorithms for computational grids Silvana," The Journal of Systems and Software, Vol. 117, 2016, Pp. 153-165.

[12] A. Hameed et al, "survey and taxonomy on energy-efficient resource allocation techniques for cloud computing systems," Computing, Vol. 98, Issue 7, 2014, pp. 751-774.

[13] S. Mishra et al, “Allocation of an energy-efficient task in the cloud using DVFS,” International Journal of Computational Science and Engineering, Vol. 18, Issue 2, 2019, pp. 154-163.

[14] B. Barzegar, H.Motameni, and A. Movaghar, "EATSDCD: A green energy-aware scheduling algorithm for parallel task-based application using clustering, duplication and DVFS technique in cloud datacenters,” Journal of Intelligent \& Fuzzy Systems, Vol. 36, No. 6, 2019, pp. 5135-5152. 
[15] M. Sharma and R. Garg, "HIGA: Harmony-inspired genetic algorithm for rack - aware energy-efficient task scheduling in cloud data centers,” Engineering Science and Technology, an International Journal, 2019, HTTP: //doi.org/10.1016/j.jestch.2019.03.009.

[16] L. Mao et al, "A multi-resource task scheduling algorithm for energy-performance tradeoffs in green clouds," Sustainable Computing: Informatics and Systems, Vol. 19, 2018, Pp. 233- 241

[17] M. Kumar and S. Sharma, "PSO-COGENT: Cost and energy-efficient scheduling in the cloud environment with deadline constraint," Sustainable Computing: Informatics and Systems, Vol. 19, 2018, Pp. 147-164.

[18] F. Fernandes et al, "A Virtual Machine Scheduler Based on CPU and I/O-Bound Features for Energy-Aware in High-Performance Computing Clouds,” Computers and Electrical Engineering, Vol. 56, 2016, pp. 854-870

[19] Chen Li, Lisu Huo, and Huangke Chen, "Real-Time Workflows Oriented Hybrid Scheduling Approach with Balancing Host Weighted Square Frequencies in Clouds” IEEE Access, DOI: 10.1109/ACCESS.2019.2955013

[20] H. Yuan et al, "Biobjective Task Scheduling for Distributed Green Data Centers," IEEE Transactions on Automation Science and Engineering. doi: 10.1109/TASE.2019.2958979, online Jan.2020.

[21] H. Yuan, et al., "Spatial Task Scheduling for Cost Minimization in Distributed Green Cloud Data Centers," IEEE Transactions on Automation Science and Engineering, Vol. 16, No. 2, April 2019, pp. 729-740.

[22] H. Yuan and H. Liu, "Revenue and Energy Cost-optimized Biobjective Task Scheduling for Green Cloud Data Centers," IEEE Transactions on Automation Science and Engineering, DOI: 10.1109/TASE.2020.2971512, online, Jan. 2020.

[23] D. Jiang et al, "An optimization-based robust routing algorithm to energy-efficient networks for cloud computing," Telecommunication Systems, 2016, Vol. 63, Issue 1, pp. 89- 98

[24] M. Sayadnavard, A. Haghighat, and A. Rahmani, “A reliable Computing: Informatics and Systems, Vol. 19, 2018, Pp. 185-203.

[25] M. Yavari, Akbar G. Rahbar, and M. Fathi, “Temperature and energy-aware consolidation algorithms in cloud computing,” Journal of Cloud Computing: Advances, Systems, and Applications, Vol. 8, Issue 1, 2019, Pp. 1-16.

[26] E. Arianyan, H. Taheri and S. Sharifian, "Novel Heuristics for Consolidation of Virtual Machines in Cloud Data Centers Using MultiCriteria Resource Management Solutions,” Journal of Supercomputing, Vol. 72, 2016, pp. 688-717.

[27] S. Esfandiarpoor, A. Pahlavan, and M. Goudarzi, "Structureaware online virtual machine consolidation for datacenter energy improvement in cloud computing,” Computers and Electrical Engineering, Vol. 42, 2015, Pp 74-89. 